\title{
Motivaciones de los estudiantes universitarios para nunca realizar actividades físico-deportivas de tiempo libre. El caso de la Universidad de Guadalajara \\ Reasons why the university students do not do any physical activity during their free time. In specific the case of the University of Guadalajara
}

\author{
*Gabriel Flores Allende, **Francisco Ruiz Juan \\ *Universidad de Guadalajara (México), **Universidad de Murcia (España)
}

\begin{abstract}
Resumen: Varias han sido las investigaciones para conocer los motivos por el cual el alumnado universitario realiza o a ha abandonado la práctica de actividad físico-deportiva de tiempo libre. Sin embargo, han indagado las barreras en aquellos que no han participado nunca en dicha actividad, debido a las repercusiones negativas que genera la inactividad fisica. Sobre una población de 65.700 estudiantes de Educación Superior de la Universidad de Guadalajara México, se ha realizado un muestreo polietápico estratificado con afijación proporcional, resultando un muestra de 1.207 sujetos, lo que significa un margen de error de $\pm 3 \%$ y un nivel de confianza del $95,5 \%$. Para la recogida de datos se ha utilizando un cuestionario estandarizado aplicándose de forma auto-administrada. Entre los principales resultados, destacar qué, los sujetos inactivos mencionan como principal barrera para ser sedentario el no tener aptitudes para el deporte, la falta de tiempo, la flojera o pereza y la carencia instalaciones deportivas cerca del hogar. Siendo similares a los que afectan a otras investigaciones en poblaciones universitarias. Además, realizar estudios en algún centro temático o regional, pertenecer a uno u otro Centro Universitario no influye o determina los motivos por el cual, los estudiante no han practicado nunca actividades físico-deportivas en su tiempo libre. Siendo únicamente sedentarios, los alumnos que realizan estudios de licenciatura. Situación que obliga a poner mayor atención a este grupo de sujetos que en caso de no adherirse a la práctica, incrementara las posibilidades de mortalidad, inhabilidad y morbilidad.
\end{abstract}

Palabra clave: motivaciones, universitarios, inactividad física.

\begin{abstract}
There has been several investigations that try to explain why the university students barely do exercise or most of them have dropped all the outdoor activities during their free time. However, this investigations have gone beyond the walls specially with the students that never do any activity, the reason of this, is due to all the side effects that the physical inactivity has. From the University of Guadalajara in Mexico, a 65.000 college student population, it was taken a sample of 1.207 individuals. The sampling procedure for this research was stratified polietapic with proportional affixation; which resulted on a +3 percent error margin and a trusted level of 95.5 percent. In order to collect the results of the poll, it was used a standard questionnaire applied in a auto-administrated way. Among the main results, it is important to highlight that the students that do not do any exercise emphasis that the reason of the lack of any activity is because they do not have the abilities to practice any sport, lack of free time, laziness or lack of sportive facilities near to their homes. These results are similar and have affected other investigations in the same manner the university students. Furthermore, the fact that a student studies in a thematic or regional center, or belong to a different university center does not affect or shows the reasons why the students do not do any physical activity during their free time. Being only the sedentary, the students that are studying a degree. This situation obligates us to emphasis and to pay more attention to this group of students, because in case of continuing without any physical activity, this could increase the rate of mortality, inability and morbidity.
\end{abstract}

Key words: motivations, college students, physical inactivity.

\section{Introducción}

Uno de los aspectos importantes para realizar práctica de actividad físico-deportiva de tiempo libre, es la motivación por la que se participa en ella. Situación que ha conducido a expertos investigadores (Markland \& Hardy, 1993; Masachs, Puente \& Blasco, 1994; Ruiz Juan 2000; Torre, Carrasco \& Medina, 2000; Castillo Viera, Vera Torrejón \& SáenzLópez, 2004; Gómez López, 2005; Kilpatrick, Hebert \& Bartholomew, 2005; Sanz Arazuri 2005; Madanat \& Merril, 2006), a conocer las razones que llevan a los estudiantes universitarios a iniciar y mantener la práctica del ejercicio físico y el deporte. Identificando tales factores, se podrán diseñar programas más eficaces para motivar la continuidad de la práctica durante la juventud (Madanat \& Merril, 2006), prolongarla a la edad adulta (Caspersen, Pereira \& Curran, 2000; Buckworth, 2001) y evitar su declinación.

También ha resultado trascendental analizar las causas que han influido en los sujetos, que luego de haber sido activos durante algún tiempo, han abandonado la práctica de actividad físico-deportiva de tiempo libre (Ruiz Juan, 2000; Bray \& Born, 2004; Castillo Viera et al., 2004; Gómez López, 2005; Kilpatrick et al., 2005; Sanz Arazuri, 2005). Con la intensión de buscar estrategias más efectivas, que promuevan la reincorporación de los jóvenes a la práctica (Wallace,

Fecha recepción: 11-08-08 - Fecha envío revisores: 11-08-08 - Fecha de aceptación: 25-10-08 Correspondencia: Gabriel Flores Allende

Facultad de Ciencias del Deporte. Universidad de Murcia.

C/ Argentina, s/n. 30720 Santiago de la Rivera- San Javier. Murcia. España.

Email: gflores@um.es
Buckworth, Kirby \& Sherman, 2000) y evitar la aparición de algunos padecimientos (Leslie, Sparling \& Owen, 2001) que tiempo atrás afectan principalmente a la adulta, y que en actualidad han comenzado a afectar a estos sectores de la población.

Sin embargo, pocos son los estudios que han revisado la relación de la actividad física y los comportamientos de los estudiantes inactivos. Siendo necesario profundizar en estos sujetos, debido a las repercusiones negativas que genera ser sedentario.

La inactividad física, es señalada como una de las diez causas que conducen a la mortalidad y la discapacidad, provocando que un gran número de personas de todas las edades, hombres y mujeres, tengan un bajo nivel óptimo de salud, capacidad funcional y de independencia (Benaziza, 1998; OMS, 2002a; Jacoby, Bull \& Neiman, 2003). A esta, se le atribuyen grandes pérdidas millonarias al estado, contribuyentes, aseguradoras y empresarios, debido a los altos costos que se derivan, y qué de igual, manera afectan a la productividad(Garret, Brasure, Schmitz, Schultz \& Humber, 2004; Chenoweth \& Leutzinger 2006). Se calcula que la carga mundial de morbilidad para el año 2020 , el $70 \%$ de las muertes serán atribuibles a la inactividad física (OMS, 2002b).

Con respecto a lo observado, resulta ineludible conocer las causas por las cuales, los jóvenes nunca han experimentado las sensaciones de realizar práctica de actividad físico-deportiva durante su tiempo libre.

Ruiz Juan (2000), en su tesis doctoral sobre los comportamientos, motivaciones y demanda de actividades físico-deportivas del alumnado Almeriense de enseñanza secundaria post obligatoria y de la Universidad de Almería, observa que las barreras que afectan la adherencia de los jóvenes universitarios a la práctica de actividad físico-deportiva de tiempo 
libre, son similares entre los estudiantes de primer y segundo ciclo. El estudiantado alude que la falta de tiempo y la pereza o la desgana, han sido los principales motivos para ser inactivos. Seguido a estos, ambos ciclos coinciden que el cansancio provocado por las actividades académicas o laborales son otras barreas para no participar o porque simplemente no les gusta.

En relación con esteúltimo planteamiento, parece pertinente exponer los resultados de Gómez López (2005) al analizar, en la Universidad de Almería en el curso académico 2000-2001, la evolución de los motivos para no haber realizado nunca práctica de actividad físico-deportiva el alumnado de primer ciclo, transcurridos cinco años (1996-1997), en contraste con los datos Hernández Rodríguez (2001), y en segundo ciclo, tres años después (1998-1999), en relación con las aportaciones de Ruiz Juan (2000). En dicha investigación se confirma que la falta de tiempo, junto a la pereza y la desgana fueron las principales razones para la mayoría de los estudiantes del curso académico 96-97, así como para el 01-02. Con respecto a los alumnos de segundo ciclo, este autor encuentra que los motivos más importantes al cabo de tres años, a la hora de asumir una conducta inactiva siguen siendo los mismos, en primer lugar los alumnos mencionan que la práctica de actividad física no les gusta, seguido por la falta de tiempo, la pereza y desgana y, por salir muy cansado del trabajo o del estudio.

Resultados similares son obtenidos por Sanz Aranzuri (2005), quien menciona que los motivos temporales como la falta de tiempo, es la razón citada por la mayoría de los estudiantes inactivos de la Universidad de la Rioja. Ocurriendo de igual manera con la pereza, considerada por esta investigadora como motivo de bajo interés. Resalta que el alumnado atribuye su inactividad al cansancio que provoca el trabajo o el estudio.

Se puede observar que los jóvenes suelen tener similares razones por la cual, nunca han participado en actividades físico-deportivas durante su tiempo libre, mismas que se mantienen con el paso del tiempo, situación que resulta preocupante, puesto que estos sectores tan llenos de energía y una vida por delante, carezcan de tiempo para la práctica, además de flojera o pereza que les causa participar en la misma.

Por lo tanto, percatándonos de las consecuencias y repercusiones negativas que trae consigo la inactividad física, consideramos necesario analizar en el alumnado universitario sedentario, los motivos por el cual nunca han participado en actividades físico-deportivas de tiempo libre. Para ello, nos planteamos los siguientes objetivos:

1. Conocer cuales son los motivos que han llevado a los estudiantes de Educación Superior de la Universidad de Guadalajara, a no haber practicado nunca actividades físico-deportivas durante su tiempo libre.

2. Indagar si las principales barreras señalados por los alumnos de la Universidad de Guadalajara, son similares a los que figuran en otras poblaciones universitarias.

3. Observar si los motivos mencionados para no haber participado nunca en actividades físico-deportivas, varían en función del centro temático o regional de la Universidad de Guadalajara, por sede del centro universitario y en la oferta académica en grado superior.

\begin{tabular}{|c|c|c|}
\hline Centro Universitario & Población (N) & $\begin{array}{c}\text { Muestra } \\
\text { (n) }\end{array}$ \\
\hline Centro Uni versitario de Arte, Arquitectura y Diseño & 4.832 & 83 \\
\hline Centro Universitario de Ciencias Biológicas y Agropecuarias & 2.587 & 44 \\
\hline Centro Universitario de Ciencias Económicas y Administrativas & 13.450 & 225 \\
\hline Centro Universitario de Ciencias Exactas e Ingenierías & 11.893 & 202 \\
\hline Centro Uni versitario de Ciencias de la Salud & 6.598 & 112 \\
\hline Centro Universitario de Ciencias Sociales y Humanidades & 7.602 & 128 \\
\hline Centro Uni versitario de los Altos & 1.561 & 38 \\
\hline Centro Uni versitario de la Ciénega & 4.268 & 93 \\
\hline Centro Universitario de la Costa & 4.435 & 95 \\
\hline Centro Universitario de la Costa Sur & 2.261 & 51 \\
\hline Centro Uni versitario de Lagos de Moreno & 1.564 & 35 \\
\hline Centro Uni versitario del Norte & 664 & 14 \\
\hline Centro Uni versitario del Sur & 2.678 & 58 \\
\hline Centro Universitario de los Valles & 1.307 & 29 \\
\hline Total & 65.700 & 1.207 \\
\hline
\end{tabular}

\begin{tabular}{|c|c|c|}
\hline \multicolumn{3}{|c|}{ Tabla 2. Motivos por los cuales Nunca han practicado actividades fisico-deportivas los } \\
estudiantes de Educación Superior de la Universidad de Guadalajara. Frecuencias y porcentajes. \\
\hline & \multicolumn{2}{|c|}{ Motivos de No Practicar Nunca } \\
\hline & N & $\%$ \\
\hline No le veo beneficios ni utilidad & 2 & 3,4 \\
\hline No me enseñaron en la escuela & 0 & 0 \\
\hline No tengo aptitudes para el deporte & 31 & 52,5 \\
\hline Por motivos económicos & 9 & 15,3 \\
\hline Porque no tengo tiempo & 29 & 49,2 \\
\hline No hay instalaciones cerca de la casa & 19 & 32,2 \\
\hline Las instalaciones no son adecuadas & 5 & 8,5 \\
\hline Mis amigos no practicaban & 8 & 13,6 \\
\hline Mis padres no me lo inculcaron & 13 & 22,0 \\
\hline No me gusta & 16 & 27,1 \\
\hline Por motivos de salud y lesiones & 4 & 6,8 \\
\hline Flojera y/o pereza & 29 & 49,2 \\
\hline Porque nadie me apoyo para iniciarme & 9 & 15,3 \\
\hline Otros & 1 & 1,7 \\
\hline
\end{tabular}

\section{Metodología}

\subsection{Muestra}

La población objeto de estudio la constituyen los estudiantes de Educación Superior de la Universidad de Guadalajara (México), durante el ciclo escolar 2005 calendario «B». Distribuidos en los catorce centros de la red Universitaria. Para seleccionar la muestra se llevó a cabo un muestreo poliétapico estratificado con afijación proporcional (Manzano, 1998).

Teniendo en cuenta los datos de la población objeto de estudio hemos buscado que la validez de los resultados nos permita asumir un margen de error de $\pm 3 \%$ y un nivel de confianza del $95,5 \%$. Es decir, la muestra seleccionada para nuestro análisis es de 1,207 estudiantes de la Universidad de Guadalajara, de los cuales 593 son mujeres y 614 varones (tabla 1).

\subsection{Instrumento}

Dadas las características propias de la investigación, el instrumento manejado ha sido el cuestionario. Para la elaboración del cuestionario fue necesaria la delimitación del campo de estudio y determinación precisa de los objetivos que pretendemos lograr en nuestra investigación y basándonos en algunos estudios con similares características a la nuestra (Sallis, Hovell \& Hofstetter, 1992; Ruiz Juan, 2000; IPAQ, 2002; Gómez López, 2005) en diferentes campos (metodología, sociología y del ámbito de las ciencias de la actividad físico-deportiva).

Mediante un total de 66 preguntas, fueron explorados los siguientes bloques: 1) factores biológicos y demográficos, 2) factores psicológicos, cognitivos y emocionales, 3) hábitos saludables, 4) factores sociales y culturales, 5) características de las actividades físicas y deportivas y, 6) índice de actividad física en la vida cotidiana (IPAQ).

Para este trabajo, las variables objeto de estudio que analizaremos serán: motivos por los que nunca han realizado práctica de actividad fisico-deportiva de tiempo libre los estudiantes, ubicación del centro universitario en la red universitaria (temático o regional), por centro universitario sede y oferta académica en grado superior (licenciatura y técnico superior universitario).

En el presente estudio han sido seleccionados solamente los estudiantes que nunca han realizado práctica físico-deportiva en su tiempo libre $(4,9 \%)$, es decir, los inactivos.

\subsection{Procedimiento}

Los pasos que se han seguido para la afijación proporcional, en primer instancia fue por Centro Universitario, a continuación se realizó de acuerdo al nivel de estudios (licenciatura y/o técnico superior universitario) y, por último, se consideró la variable sexo, respetando en cada uno de los estratos la misma fracción del muestreo.

El trabajo de campo se realizo en los meses de octubre a diciembre de 2005, en los centros temáticos y regionales de la red universitaria, aplicándose en aulas de manera auto-administrada en presencia de un encuestador. Destinando el alumno un promedio de 20 minutos. Para el 


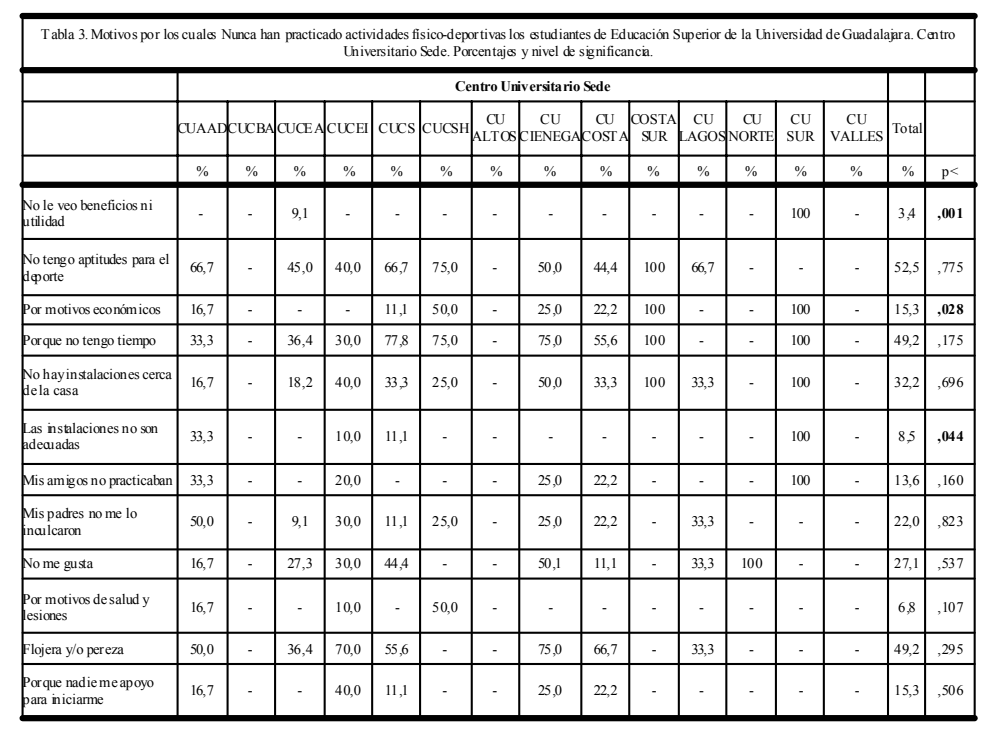

análisis de los datos utilizamos el programa informático Statistickal Package for Social Sciences (SPSS/PC versión 14.0). Hemos utilizado el Chi-cuadrado de Pearson, para observar las relaciones entre las variables tratadas en el estudio. A su vez, optamos por un estudio descriptivo procurando describir sistemáticamente hechos y características de nuestra población.

Para conocer las causas o motivos por los que nunca han realizado práctica de actividad físico-deportiva de tiempo libre, solicitamos a los estudiantes mencionaran las principales razones para no hacerlo. La pregunta, estuvo compuesta por 14 ítems. Cada ítem refleja una diferente razón o barrera por la cual el sujeto no ha participado nunca en la actividad físico-deportiva de tiempo libre.

\section{Análisis y discusión de los resultados}

3.1. Motivos por el cual no han realizado nunca práctica de actividad físico-deportiva los estudiantes Educación Superior de la Universidad de Guadalajara durante su tiempo libre

Conocer las distintas motivaciones que provocan a no llevar a cabo una práctica saludable de actividad físico-deportiva, resulta ser importante a la hora de interpretar esta conducta, permitiéndonos profundizar en este sector de la población, para intervenir con la oferta de mejores diseños, que permitan la reducción y supresión de estas barreras encontradas por los estudiantes, que les conduce a ser sedentarios.

Según los datos estadísticos, poco más de la mitad de los estudiantes inactivos, dice no haber participado nunca en actividades fisico-deportivas durante su tiempo libre, quienes perciben no tener aptitudes para la práctica del deporte (52,5\%), siendo la principal barrera. Seguida a esta, los jóvenes mencionan que la falta de tiempo y la flojera o la pereza $(49,2 \%)$, son dos barreras muy fuertes por la cual, en ningún momento de su vida han sido activos. Además, resaltan que no contar con instalaciones o infraestructura deportiva cerca a la zona de residencia $(32,2 \%)$, o simplemente porque no les gusta participar en dichas actividades (27,1\%), significa un obstáculo (tabla 2).

3.2.Principales motivos para no haber realizado nunca práctica de actividad físico-deportiva entre los jóvenes universitarios Al hablar de inactividad fisica, no podemos olvidar de las repercusiones negativas que esta genera al ser humano, tanto física (Benaziza, 1998; OMS, 2002a; Jacoby et al., 2003), como socialmente (Garret et al., 2004; Chenoweth \& Leutzinger 2006) en todo el mundo. Esto conduce a observar que causas están afectando la adherencia de los estudiantes universitarios, que a lo largo de su vida, no han participado nunca en la práctica de actividad físico-deportiva de tiempo libre. Identificando tales factores, se podrán diseñar programas eficaces que inciten a los jóvenes a ser físicamente activos, para reducir la carga mundial de morbilidad pronosticada para el año 2020 (OMS, 2002b).

En nuestro caso, se aprecia que el principal motivo por el cual los jóvenes dicen no haber participado nunca en la práctica de actividad físico-deportiva de tiempo libre, es debido a que carecen de aptitudes para realizar deporte. Esta causa difiere a los datos obtenidos por Ruiz Juan, (2000) y Gómez López (2005), en los alumnos de la Universidad de Almería y a Sanz Aranzuri (2005), en la Rioja, al encontrar en sus poblaciones diferentes barreras con respecto a la nuestra.

Para el alumnado de laUniversidadde Guadalajara, que al momento de estudio afirmó no realizar ningún tipo de actividad físico-deportiva en su tiempo libre, la falta de tiempo y, la pereza y la desgana, han sido dos barreras que han obstaculizado su participación dichas actividades. Estas razones también aparecen como las más destacadas por los inactivos de las poblaciones similares a la nuestra (Ruiz Juan, 2000; Gómez López, 2005 y Sanz Aranzuri, 2005).

Igualmente, la carencia de instalaciones cercanas al domicilio, también aparece como una de las razones de peso para ser sedentario entre los alumnos inactivos de nuestra universidad. La cual figura dentro de las cinco más citadas por los alumnos de primer ciclo de la Universidad de Almería (Gómez López, 2005) y los Universitarios de la Rioja (Sanz Arazuri, 2005), no ocurriendo así en los obtenidos por Ruiz Juan (2000), en el ciclo escolar 98/99.

Esto viene a confirmar que las razones de inactividad que afectan a nuestros estudiantes, figuran en otras poblaciones de universitarios, razón preocupante en sujetos tan llenos de energía y con futuro prometedor. Puesto que en caso de no contrarrestar estas barreras, el alumnado probablemente comience a padecero probablemente padezcan algunos males que afectan a la población, a causa de la inactividad física.

3.3. Motivos para nunca haber realizado práctica de actividad físico-deportiva de tiempo libre. Relación con la ubicación del centro universitario, la sede del centro universitario y por el nivel de estudios

Con respecto a nuestro tercer objetivo, nos planteamos la necesidad de verificar si esta variable ejerce alguna correlación significativa en relación a la ubicación del centro universitario (temático y regional), por sede del mismo y de acuerdo al nivel de oferta académica en grado superior (licenciatura y técnico superior universitario).

$\mathrm{Al}$ analizar motivos que han influido en los estudiantes para ser inactivos, con respecto a la ubicación del centro universitario, observamos que realizar estudios en algún centro temático o regional, no influye o determina en las barreras que han conducido a los estudiantes a ser inactivos en la actividad físico-deportiva de tiempo libre.

En relación al centro universitario sede, observamos que las diferencias significativas surgen en los ítems «no le veo beneficios ni utilidad» $(\mathrm{p}<.001)$, «por motivos económicos» $(\mathrm{p}<.028)$ y «las instalaciones no son adecuadas» $(\mathrm{p}<.044)$ (tabla 3$)$.

Observamos que el primer motivo (no verle beneficios ni utilidad), ha sido una barrera para ser sedentario, en alumnos de los centros universitarios de Ciencias Económicas y Administrativas $(9,1 \%$ ) y el Sur $(100 \%)$, resultando llamativo, a sabiendas de los múltiples beneficios que genera la práctica de actividad físico-deportiva de tiempo libre en la salud de los sujetos, que jóvenes universitarios mencionen esta causa para ser inactivos. Lo que obliga a poner mayor atención y difundir en estos sectores de la población, las ventajas y beneficios que supone ser fisicamente activo.

Los motivos económicos, son otros de los motivos que el alumnado universitario alude ser causante de la no participación en la práctica de actividad físico-deportiva de tiempo libre durante su vida. Esta barrera 
ha sido mencionada por los estudiantes de los centros universitarios de la Costa Sur y del Sur, en su totalidad (100\%), la mitad de los inactivos de Ciencias Sociales y Humanidades (50\%), la cuarta parte quienes estudian en la Ciénega (25\%), otros más de la Costa (22,2\%), los jóvenes de Arte, Arquitectura y Diseño (16,7\%) y en Ciencias de la Salud $(11,1 \%)$. Situación que nos hace suponer que los gastos generados por los estudios, la vivienda, los alimentos y otros más, han obligado a estos jóvenes a destinar los recursos financieros a estas actividades, resultando importante transmitir las múltiples actividades físico-deportivas que pueden ser realizadas, sin necesidad de invertir grandes cantidades de dinero para llevarlas a cabo.

El valorar que las «instalaciones deportivas no son las adecuadas» significo ser una barrera entre los estudiantes que dicen ser inactivos, razón por la cual nunca han participado en actividades físico-deportivas de tiempo libre. Probablemente, esto ocurra debido a que, estas se encontrarán en mal estado, no satisfacen las necesidades requeridas para la práctica deseada o la infraestructura es insuficiente. Por tanto, percibir que las instalaciones son inadecuadas, ha sido mencionada por todos los alumnos inactivos del Centro Universitario de Arte, Arquitectura y Diseño (33,3\%), Ciencias de la Salud (11,1\%), Ciencias Económicas y Administrativas $(10 \%)$ y del Sur $(100 \%)$, resultando llamativo que todos los sujetos sedentarios de este último campus, coinciden en mencionar este motivo para no practicar.

Por lo tanto, ser alumno de uno u otro Centro Universitario no influye o determina los motivos por el cual, los estudiantes no han practicado nunca actividades físico-deportivas de tiempo libre. Que según observamos, puede presentarse en ciertos casos, por no verle utilidad a la práctica, los problemas económicos y no contar con instalaciones adecuadas.

Cabe destacar que según los resultados estadísticos, en once de los catorce centros universitarios, los alumnos indicaron alguna causa, por la cual han sido sedentarios. Es decir, en el resto los estudiantes realizan práctica de actividad físico-deportiva de tiempo libre o la han abandonado, sin presentar caso alguno de inactividad. Esto ocurre en Ciencias Biológicas y Agropecuarias, en los Altos y los Valles.

En lo que refiere al nivel de estudios que se oferta en grado superior (licenciatura y técnico superior universitario), podemos comentar que según los datos estadísticos, ningún estudiante que realiza estudios de Técnico Superior Universitario, es sedentario o inactivo.

\section{Conclusiones}

Luego de preguntar a los estudiantes de Educación Superior de la Universidad de Guadalajara, porque causas o motivos no han participado nunca en la práctica de actividad físico-deportiva de tiempo libre, observamos que afronta un grave problema el alumnado que manifiesta ser inactivo. Situación que obliga a poner mayor atención a este grupo se sujetos que en caso de no adherirse a la actividad físico-deportiva, se incrementaran las cifras de mortalidad, morbilidad e inhabilidad.

Por otro lado, no encontramos diferencias importantes con respecto a las barreras que vienen afectando la práctica de actividad físico-deportiva en estas poblaciones, las cuales son similares con el paso del tiempo.

En tanto, las variables centro temático o regional, sede del centro universitario y oferta académica en grado superior, no influye en los motivos por el cual, el estudiantado dice ser físicamente inactivo en la práctica de actividad físico-deportiva del tiempo libre. Que al margen de esto, puede presentarse en ciertos casos en particular, no siendo determinantes.

\section{Referencias bibliográficas}

Benaziza, H. (1998). Creación de asociaciones: una clave para promover la actividad fisica para la salud. In Informe final. El deporte para todos y los retos educativos a nivel mundial (pp. 186). Barcelona.

Bray, S. y Born, H. (2004). Transition to University and Vigorous Physical Activity: Implications for Health and Psychological Well-Being. Journal of American College Health. 52, 181-188.
Buckworth, J. (2001). Exercise adherence in college students: issues and preliminary results. Quest, 53, 335-345.

Caspersen, C., Pereira, M. y Curran, K. (2000). Changes in physical activity patterns in the United States. Medicine \& Science In Sports \& Exercise, 32.

Castillo Viera, E., Vera Torrejón, C. y Sáenz-López Buñuel, P. (2004). Motivos de práctica de actividad fisica del alumnado de la universidad de Huelva. In A Coruña (Galicia).

Chenoweth, D. y Leutzinger, J. (2006). The Economic Cost of Physical Inactivity and Excess Weight in American Adults. Journal of physical activity and health. 3, (2)

Gómez López, M. (2005). La actividad fisico-deportiva en los centros almerienses de educación secundaria post obligatoria y en la Universidad de Almería. Evolución de los hábitos físico-deportivos de su alumnado. Universidad de Almería.

Garret, N., Brasure, M., Schmitz, K., Schultz, M. y Humber, M. (2004). Physical Inactivity: Direct cost to a health plan. American Journal of Preventive Medicine. 27, (4) 304-309

International Physical Activity Questionnaire. (2002). Cuestionario Internacional de Actividad Física. Versión Larga formato auto administrado-últimos 7 días para uso con Jóvenes y Adultos de Mediana edad (15-69 años).

Jacoby, E., Bull, F. y Neiman, A. (2003). Cambios acelerados del estilo de vida obligan a fomentar la actividad física como prioridad en la Región de las Américas. Revista Panamericana de la Salud, 14, 223225

Leslie, E., Sparling, P. y Owen, N. (2001). University campus settings and the promotion of physical activity in young adults: Lessons from research in Australia and the USA. Health Education. 101, (3) 116125

Kilpatrick, M., Hebert, E. y Bartholomew, J. (2005). Collage students' motivation for physical activity: Differentiating men's and women's motives for sport participation and exercise. Journal of American College Health, 54 (2) 87-94.

Madanat, H. y Merril, R. (2006). Motivational factors and stages of change for physical activity among collage students in Amman, Jordan. Promotion \& Education. 13; 3, 185-190

Masachs, M., Puente, M. y Blasco, T. (1994). Evolución de los motivos para participar en programas de ejercicio físico. Revista de Psicología del Deporte. 5, 71-80

Markland, D. y Hardy, L. (1993). The exercise motivations inventory: preliminary development and validity of a measure of individuals' reasons for participation in regular physical exercise. Personality \& Individual Differences, 15, 289-296

Organización Mundial de la Salud. (2002a). Sedentary lifestyle: a global public health problem. Organización Mundial de la Salud. 11-42006.

Organización Mundial de la Salud. (2002b). Informe sobre la salud en el mundo 2002, promover los riesgos y promover una vida sana. Organización Mundial de la Salud.

Ruiz Juan, F. (2000). Análisis diferencial de los comportamientos, motivaciones y demanda de actividades físico-deportivas del alumnado Almeriense de enseñanza secundaria post obligatoria y de la Universidad de Almería. Universidad de Granada.

Sanz Arazuri, E. (2005). La práctica fisico-deportiva de tiempo libre en universitarios. Análisis y propuestas de mejora. Servicio de publicaciones de la Universidad de la Rioja.

Sallis, J., Hovell, M. y Hofstetter, R. (1992). Predictors of adoption and maintenance of vigorous physical activity in men and women. Preventive Medicine, 21, 237-251.

Torre, G., Carrasco, L. y Medina, J. (2000). Investigación sobre porque los estudiantes universitarios practican deporte. El caso de la Universidad de Granada. Revista Motricidad, 6; 95-105

Wallace, L.S., Buckworth, J., Kirby, T.E. y Sherman, W.M. (2000). Characteristics of

Exercise Behavior among College Students: Application of Social Cognitive Theory to Predicting Stage of Change. Preventive Medicine 31, 494505 